\title{
Neutral and charged excitations in carbon fullerenes from first-principles many-body theories
}

\author{
Murilo L. Tiago, ${ }^{1}$ P. R. C. Kent,${ }^{1}$ Randolph Q. Hood, ${ }^{2}$ and Fernando A. Reboredo ${ }^{1}$ \\ ${ }^{1}$ Oak Ridge National Laboratory, Oak Ridge, TN, 37831 \\ ${ }^{2}$ Lawrence Livermore National Laboratory, Livermore, California, 94550
}

(Dated: October 22, 2018)

\begin{abstract}
We investigate the accuracy of first-principles many-body theories at the nanoscale by comparing the low energy excitations of the carbon fullerenes $\mathrm{C}_{20}, \mathrm{C}_{24}, \mathrm{C}_{50}, \mathrm{C}_{60}, \mathrm{C}_{70}$, and $\mathrm{C}_{80}$ with experiment. Properties are calculated via the GW-Bethe-Salpeter Equation (GW-BSE) and diffusion Quantum Monte Carlo (QMC) methods. We critically compare these theories and assess their accuracy against available photoabsorption and photoelectron spectroscopy data. The first ionization potentials are consistently well reproduced and are similar for all the fullerenes and methods studied. The electron affinities and first triplet excitation energies show substantial method and geometry dependence. These results establish the validity of many-body theories as viable alternative to density-functional theory in describing electronic properties of confined carbon nanostructures. We find a correlation between energy gap and stability of fullerenes. We also find that the electron affinity of fullerenes is very high and size-independent, which explains their tendency to form compounds with electron-donor cations.
\end{abstract}

PACS numbers: 78.66.Tr,73.22.-f,78.20.Bh,78.40.Ri 


\section{INTRODUCTION}

Carbon fullerenes form a remarkable series of molecules whose physical and chemical properties are relatively well known. Their properties can be controlled through size, shape, and structure|1]. They can be functionalized, doped with endohedral or exohedral atoms, or polymerized [3]. $\mathrm{C}_{60}$ is the most stable and well-studied molecule in the series. It has been widely used e.g. in the design of efficient organic photovoltaic cells [4, 5] and as precursor in the growth of carbon nanotubes and nanopeapods [6, 7]. The strength of carbon $s p^{2}$ bonds makes fullerenes extremely stable, and the ability of carbon to form bonds with different hybridization levels makes possible the existence of both hexagonal and pentagonal rings and hence a rich variety of sizes and shapes[1, 3]. In fact, carbon is the main constituent of a wide range of nanostructures from diamondoids to organic molecules, including fullerenes and nanotubes. It is likely that nano-electronic devices with desired properties can be created through intentional selection of specific carbon nanostructures [1, 8]. In order to systematically design such devices it is necessary to develop a thorough understanding of the electronic and optical properties of the nanostructure, either isolated or in a functionalized medium. Detailed knowledge of the electronic structure of fullerene molecules is the key to explore several properties of those systems. As an example, the conductance of fullerenebased junctions depends crucially on the relative energy of electronic orbitals on the molecule with respect to the chemical potential on the leads [9].

Experimental characterization is often hindered at the nanoscale and therefore theory plays an increasingly important role in nano-science as a guide for experimental interpretation. While it is often claimed that theory can be used to design nanomaterials with targeted properties, for theory to have predictive power it is crucial to validate it in those precious cases where the comparison with experiment can be made. Carbon compounds including nanotubes, [10, 11] adamatanes and fullerenes are among the few nanomaterials that have been characterized in terms of structure and composition.

In this paper we apply the two most accurate approaches for calculating electronic/optical properties, the GW-Bethe Salpeter equation (GW-BSE) approach [12, 13] and diffusion quantum Monte Carlo (QMC)[14]. These methods are complementary: the GW-BSE approach is based primarily on approximate Green's functions and in-principle yields the entire optical spectrum, while QMC uses a stochastic sampling approach that is in-principle exact 
for the lowest energy excitation of each symmetry[14]. Due to recent algorithmic advances and a favourable scaling with system size, both of these methods can provide the optical properties of nanostructures with one hundred atoms or more using existing parallel computers [15, 16]. While GW-BSE and QMC have previously shown good agreement for small molecules [12, 13, 16, 17], this is the first comprehensive study of these methods for these much larger yet prototypical nanostructures. Owing to the complexity involved in both QMC and GW-BSE when applied to electronically confined systems, not much is known about the accuracy of either theory in confined nanostructures.

We study a range of fullerenes $\left(\mathrm{C}_{20}, \mathrm{C}_{24}, \mathrm{C}_{50}, \mathrm{C}_{60}, \mathrm{C}_{70}\right.$, and $\left.\mathrm{C}_{80}\right)$, with the aim of answering these questions: (1) Do these methods accurately predict the optical and electronic properties, particularly for the best experimentally characterized $\mathrm{C}_{60}$ molecule? (2) What are the trends in the physical properties across the fullerene series? (3) Where are improvements in each method required?

\section{METHOD}

Fullerenes are routinely produced in a wide range of sizes, from $\mathrm{C}_{20}$ to $\mathrm{C}_{100}$ or larger, and many isomers have been identified [18]. The existence of several low-energy isomers for each molecular size poses a difficulty in modeling fullerenes. For each computed size we have chosen high-symmetry structures that satisfy the isolated pentagon rule [1] as closely as possible. Figure 1 depicts the isomers investigated here, with the corresponding point groups. The atomic coordinates were obtained by minimizing the total energy within normconserving pseudopotential calculations using DFT and the Perdew-Burke-Ernzerhof (PBE) functional [19]. Choices of grid point separation, energy cutoffs and supercell sizes were such that Kohn-Sham eigenvalues of bound orbitals are converged to $0.02 \mathrm{eV}$ or better and bond lengths to $1 \%$ or better. Atom coordinates are included in the supporting information accompanying this paper[2].

Owing to its simplicity, density-functional theory (DFT) has been used to predict the electronic properties of nanostructures. Nevertheless, it has two major deficiencies: (1) It can only describe the energy of optical excitations through ad hoc approaches such as constrained DFT [20]; and (2) it is not possible to systematically improve the description of exchange and correlation effects within DFT because the exact exchange-correlation functional cannot 
be calculated in closed form [20]. In contrast, many-body approaches can be systematically improved, at the expense of increased numerical complexity.

In the framework of the GW approximation [13], electron self-energies are calculated in terms of the Green's function $G$ and the screened Coulomb interaction $W$ as:

$$
\Sigma(1,2)=i G(1,2) W_{0}(1,2)
$$

where screening is commonly evaluated in the random-phase approximation (RPA). We can modify Eq. (11) by replacing the RPA with time-dependent DFT with an adiabatic, local-density kernel (TDLDA). In order to retain Feynman diagrams with the same order, we add a properly symmetrized vertex term together with the TDLDA screened Coulomb interaction [15]:

$$
\Sigma(1,2)=i \int \mathrm{d}(3) G(1,3) W(3,2) \Gamma(1,2 ; 3)+\text { symm. . }
$$

At its lowest level of approximation, the Green's function and the screened Coulomb interaction above are calculated within DFT [13]. Further improvement in the theory is attained by imposing self-consistency among the self-energy $\Sigma$, Green's function $G$ and screened Coulomb interaction $W$ [13] We impose self-consistency in an iterative scheme. In the first iteration, we calculate the self-energy using DFT electronic orbitals and Eq. (2). In subsequent iterations, we use a scissors operator to correct the energy of DFT orbitals, according to the self-energy calculated in the previous iteration. We stress the fact that the scissors operator is constructed using information from the previous iteration only. No free parameters are involved. We stop when electron affinity and ionization potential are converged to within $0.02 \mathrm{eV}$. Usually, this is reached in less than five iterations. Tests performed on smaller molecules $\left(\mathrm{Cl}_{2}\right.$, benzene, $\mathrm{CO}, \mathrm{SiH}_{4}$ ) indicate that going behond the scissors operator method would improve the final results by $0.1 \mathrm{eV}$ or less. Scissors operators have been used extensively in the context of electronic band structure of bulk semiconductors (see Ref. 13 and references therein). In the following, we refer to Eqs. (11) and (2) as $\mathrm{GW}_{0}$ and $\mathrm{GW}_{f}$ approximations respectively. We denote by scGW $\mathrm{G}_{f}$ approximation the self-consistent solution of Eq. (2), where self-consistency is imposed through a scissors operator. After the quasi-particle orbitals are determined, we calculate optical excitations by diagonalizing the Bethe-Salpeter equation for electrons and holes following standard methodologies 
[12, 15, 17]. The BSE method has been used successfully to predict excitation energies of organic molecules [15], CdSe nanocrystals [21] and hydrogenated silicon clusters [17].

In the QMC methodology (for a review, see Ref. [14]), excitation energies are obtained via the difference in total energy between the ground state and individually constructed excited states. We use the diffusion Monte Carlo (DMC) technique with trial wave-functions consisting of a single Slater determinant of single-particle orbitals multiplied by a Jastrow correlation function [22]. The most significant approximation is the fixed-node approximation, whereby the Fermion nodes of a trial wave-function are used in place of the exact nodes, introducing a variational error. Although DMC calculations for large systems additionally require the use of pseudopotentials, for carbon-based systems this approximation is secondary. We evaluate the pseudopotentials within the locality approximation. A test calculation for $\mathrm{C}_{20}$ using a variational pseudopotential evaluation[23] found a global shift of $0.8 \pm 0.1 \mathrm{eV}$ but identical energy differences to within $\pm 0.1 \mathrm{eV}$, demonstrating that this error is small in the fullerenes. In practice, the accuracy of the results obtained for excitations is solely determined by the accuracy of the ground and excited state nodal surfaces. In contrast, the GW calculations are dependent on the detailed shape of the Kohn-Sham orbitals and the initial Kohn-Sham eigenvalues. Given exact nodes for the ground state and for the first excited state of a given symmetry, DMC yields in-principle exact results[14]. In general, there are additional symmetry related qualifications [24]. In our calculations we use the Kohn-Sham orbitals from the ground state DFT calculation in the Slater determinant to effectively define the nodal surface of the trial wavefunction. DMC was used recently to calculate excitation energies and ionization energies of diamondoids yielding very good results [25].

To make comparisons between GW-BSE and QMC on an equal footing we used the same norm conserving pseudopotential in each set of calculations. The initial QMC wave-functions were obtained from a local-density approximation (LDA) DFT calculation, while for GW calculations we additionally investigated the PBE[19] gradient corrected functionals. $\mathrm{GW}_{f}$ electron affinities and vertical ionization potentials obtained using the LDA are higher than the ones obtained using PBE by a fraction of electron-volt: $0.1 \mathrm{eV}$ in $\mathrm{C}_{60}$ and $0.3 \mathrm{eV}$ in $\mathrm{C}_{20}$. The impact of replacing the LDA with PBE on Kohn-Sham eigenvalues is even smaller: less than $0.05 \mathrm{eV}$. In view of these small differences we concentrate on LDA-derived results. 


\section{RESULTS}

We have investigated two isomers of $\mathrm{C}_{80}$. DFT calculations have already indicated that $\mathrm{C}_{80}$ exists in a large number of isomers, where the oval shaped structure (point group $\mathrm{D}_{5 \mathrm{~d}}$ ) is the most stable one [26, 27]. Our DFT calculations predict that the icosahedral isomer $\left(\mathrm{I}_{h}\right)$ is more energetic than the $\mathrm{D}_{5 \mathrm{~d}}$ isomer by $0.8 \mathrm{eV}$, confirming earlier calculations [26]. This difference increases to $1.74 \pm 0.10 \mathrm{eV}$ in QMC.

Table 1 shows the vertical ionization potential (IP) calculated within the GW and QMC theories. The ionization potential was also calculated using DFT only, as the difference in total energy between the neutral and the charged molecules (" $\triangle \mathrm{SCF}-\mathrm{DFT}$ ") column. The IP is approximately $7.5 \mathrm{eV}$ for all of the theories, with no well defined trend in IP with size apart from a slow decrease with increasing number of atoms. The analyzed structures have different curvatures, caused by the different positions of pentagonal rings from molecule to molecule. Therefore, geometry and symmetry become important factors in determining the ionization potential. A classical electrostatic model based simply on a charged hollow sphere can only predict qualitatively the ionization potential of fullerenes [28, 29]. Except for $\mathrm{C}_{60}$ and $\mathrm{C}_{70}$, the $\mathrm{QMC}$ ionization potential is lower than the $\mathrm{GW}_{f}$ ionization potential. The discrepancy between the two approaches is around $0.2 \mathrm{eV}$, close to the numerical accuracy

associated with each theory [44]. Except for $\mathrm{GW}_{0}$ all the methods predict IPs close to experiment. Self-consistency in the GW method is found to give very small improvement on the already accurate IP. Table $\Pi$ also shows that the $\mathrm{GW}_{f}$ approximation predicts an IP much more accurate than the $\mathrm{GW}_{0}$ approximation. Therefore, including vertex corrections and polarizability effects beyond RPA are an improvement on the theory, confirming similar observations in benzene and naphthalene molecules [15].

Ultra-violet photoelectron spectroscopy (UPS) can determine not only the first ionization potential but also higher ionization energies, thus giving direct access to the electronic structure of the material. Lichtenberger and collaborators [30] have identified five ionization bands in the $\mathrm{C}_{60}$ spectrum. Table II shows the assignment of the ionization bands and comparison between measured and calculated vertical ionization energies. Higher-order ionization energies can only be calculated within DFT if a minimization constraint is used. Calculation results presented on column "CDFT" on Table I were obtained by minimizing the total energy of the cation $\mathrm{C}_{60}^{+}$with the constraint that the ejected electron occupied a 
TABLE I: Vertical ionization potentials calculated from DFT, QMC and GW theories. Error bars in $\mathrm{QMC}$ values are indicated between parentheses. All energies in $\mathrm{eV}$.

\begin{tabular}{ccccccc}
\hline \hline & $\Delta$ SCF-DFT & QMC & GW $_{0}$ & GW $_{f}$ & scGW $_{f}$ & Exp. \\
\hline $\mathrm{C}_{20}$ & 7.31 & $7.27(11)$ & 7.99 & 7.35 & 7.41 & \\
$\mathrm{C}_{24}$ & 7.77 & $7.70(10)$ & 8.49 & 7.86 & 7.81 & \\
$\mathrm{C}_{50}$ & 7.29 & $7.29(14)$ & 7.97 & 7.33 & 7.35 & $7.61^{a}$ \\
$\mathrm{C}_{60}$ & 7.61 & $7.86(21)$ & 8.22 & 7.70 & 7.86 & $7.6^{b}$ \\
$\mathrm{C}_{70}$ & 7.54 & $7.69(12)$ & 8.12 & 7.53 & 7.45 & $7.47^{c}$ \\
$\mathrm{C}_{80}\left(\mathrm{D}_{5 d}\right)$ & 6.67 & $6.30(10)$ & 7.24 & 6.59 & 6.65 & $6.84^{a}$ \\
$\mathrm{C}_{80}\left(\mathrm{I}_{h}\right)$ & 6.86 & $6.91(10)$ & 7.45 & 6.90 & 6.95 & \\
\hline average error & -0.10 & -0.09 & 0.51 & -0.09 & -0.05 & \\
root mean square error & 0.18 & 0.36 & 0.52 & 0.20 & 0.21 & \\
\hline \hline
\end{tabular}

${ }^{a}$ Ref. 18. ${ }^{b}$ Ref. $30{ }^{c}$ Ref. 38

specific molecular orbital (HOMO, HOMO-1, or deeper orbitals). Whereas constrained DFT predicts very accurately the first two ionization energies, it increasingly underestimates the higher ionization energies. The discrepancy seems to be more than $1 \mathrm{eV}$ in the ionization band at 12.4 to $13.8 \mathrm{eV}$. In contrast, the self-consistent $\mathrm{GW}_{f}$ approximation predicts ionization energies no more than $0.35 \mathrm{eV}$ away from the experimental data over the same energy range.

The electron affinity (EA), shown in Table III is also predicted differently by QMC and the various levels of GW theory. The former theory underestimates the electron affinity by as much as $1 \mathrm{eV}$ relative to the latter one. Inclusion of vertex corrections (from $\mathrm{GW}_{0}$ to $\mathrm{GW}_{f}$ ) reduces the electron affinity by approximately $0.6 \mathrm{eV}$. Inclusion of self-consistency (from $\mathrm{GW}_{f}$ to $\mathrm{scGW} f$ ) increases the self-energy by a further $0.5 \mathrm{eV}$, bringing the calculated results in very good agreement with experimental data and with $\triangle \mathrm{SCF}$-DFT predictions. The electron affinity is found to be around 2 to $4 \mathrm{eV}$. All theories employed in this work predict that $\mathrm{C}_{20}$ has the smallest electron affinity whereas $\mathrm{C}_{80}\left(\mathrm{I}_{h}\right)$ has the largest electron affinity. $\mathrm{C}_{60}$ is well known to be easily ionizable [1, 3, 31, 32]. The present results also show that less stable fullerenes are easily ionizable as well, which opens the path to functionalize fullerenes of different sizes. 
TABLE II: Orbital assignment and vertical ionization energy for the highest occupied molecular orbitals in $\mathrm{C}_{60}$. Column "C-DFT" shows constrained DFT results. Columns $\mathrm{GW}_{0}$ and $\mathrm{GW}_{f}$ are non-self-consistent GW calculations, with and without vertex corrections respectivelly. Column $\mathrm{scGW}_{f}$ includes self-consistency and vertex corrections. Experimental data quoted from Ref. 30. Experimental data spanning multiple rows indicate ionization bands that could not be resolved. All energies in $\mathrm{eV}$.

\begin{tabular}{cccccc}
\hline \hline & $\mathrm{C}-\mathrm{DFT}$ & $\mathrm{GW}_{0}$ & $\mathrm{GW}_{f}$ & $\mathrm{scGW}_{f}$ & Exp. \\
\hline $\mathrm{h}_{u}$ & 7.61 & 8.22 & 7.70 & 7.86 & 7.6 \\
\hline $\mathrm{g}_{g}$ & 8.78 & 9.33 & 8.85 & 9.23 & 8.95 \\
$\mathrm{~h}_{g}$ & 8.90 & 9.42 & 8.93 & 9.25 & \\
\hline $\mathrm{h}_{u}$ & 10.47 & 11.93 & 11.40 & 11.93 & $10.82-11.59$ \\
$\mathrm{~g}_{u}$ & 10.50 & 11.00 & 10.51 & 11.07 & \\
$\mathrm{t}_{2 u}$ & 11.03 & 11.46 & 11.02 & 11.6 & $12.43-13.82$ \\
\hline $\mathrm{h}_{g}$ & 10.79 & 12.19 & 11.65 & 12.2 & 13.36 \\
$\mathrm{~g}_{u}$ & 11.79 & 13.23 & 12.73 & 13.76 & 13.04 \\
$\mathrm{t}_{1 g}$ & 12.28 & 13.60 & 13.11 & 12.32 & \\
$\mathrm{~h}_{g}$ & 12.33 & 12.74 & & \\
\hline \hline
\end{tabular}

One important result of this work is that $\mathrm{GW}_{0}$ and $\mathrm{GW}_{f}$ are shown to be consistent in the "electronic gap", defined as the difference between ionization potential and electron affinity. This definition of electronic gap is the closest analogue of band gap to isolated molecules [13]. Although $\mathrm{GW}_{0}$ and $\mathrm{GW}_{f}$ predict different values for IP and EA, the differences cancel out in the electronic gap. The reason for this cancellation is that contributions arising from LDA screening, such as the vertex $\Gamma$, have similar magnitude when evaluated at the highest occupied molecular orbital (HOMO) and at the lowest unoccupied molecular orbital (LUMO). As a consequence of this cancellation, the contributions from LDA screening are often neglected in calculations of electronic gap in crystals [13]. In molecular systems, these contributions are not negligible because they affect IP and EA separately [15, 33]. Selfconsistency has been found to systematically increase the electronic gap compared to nonself-consistent GW methodologies in a variety of different materials [13, 34]. Our calculations confirm the same behavior in finite systems, and they also indicate that self-consistency 
TABLE III: Electron affinity, or vertical detachment energy, calculated from DFT, QMC and GW theories. Error bars in QMC values are indicated between parentheses. All energies in eV.

\begin{tabular}{ccccccc}
\hline \hline & $\Delta \mathrm{SCF}-\mathrm{DFT}$ & $\mathrm{QMC}$ & $\mathrm{GW}_{0}$ & $\mathrm{GW}_{f}$ & $\mathrm{scGW}_{f}$ & Exp. \\
\hline $\mathrm{C}_{20}$ & 2.17 & $1.76(11)$ & 3.55 & 2.92 & 2.36 & $2.25^{a}$ \\
$\mathrm{C}_{24}$ & 3.04 & $2.57(11)$ & 4.19 & 3.55 & 2.88 & \\
$\mathrm{C}_{50}$ & 3.73 & $3.52(14)$ & 4.75 & 4.12 & 3.73 & $<3.10^{b}$ \\
$\mathrm{C}_{60}$ & 2.94 & $2.23(19)$ & 3.87 & 3.33 & 2.98 & $2.69^{c}$ \\
$\mathrm{C}_{70}$ & 2.96 & $2.46(11)$ & 3.98 & 3.35 & 2.83 & $2.76^{d}$ \\
$\mathrm{C}_{80}\left(\mathrm{D}_{5 d}\right)$ & 3.46 & $3.25(10)$ & 4.62 & 3.91 & 3.88 & $3.70^{b}$ \\
$\mathrm{C}_{80}\left(\mathrm{I}_{h}\right)$ & 3.98 & $3.90(11)$ & 5.17 & 4.61 & 4.38 & \\
\hline average error & 0.03 & -0.42 & 1.25 & 0.53 & 0.16 & \\
root mean square error & 0.20 & 0.43 & 1.16 & 0.56 & 0.18 & \\
\hline \hline
\end{tabular}

${ }^{a}$ Ref. 39. ${ }^{b}$ Ref. 40. ${ }^{c}$ Ref. 31. ${ }^{d}$ Ref. 32.

affects the energy of unoccupied orbitals more than of occupied orbitals. Self-consistency could be more important in fullerenes than in most small molecules studied so far [15, 17, 21, 35] because of the narrower HOMO-LUMO gap.

There is an ongoing debate in the literature concerning the importance of vertex corrections [13, 15, 33]. The $\mathrm{GW}_{f}$ approximation has vertex corrections included in two ways: explicitly through the function $\Gamma$ in Eq. (2), and implicitly through the screened Coulomb interaction (see e.g. Eq. 17 and 18 in Ref. [15]). We have observed that most of the difference between $\mathrm{GW}_{f}$ and $\mathrm{GW}_{0}$ calculation results originates from the explicit contribution rather than the implicit contribution. Matrix elements of the "explicit vertex contribution", defined as $\Delta \Sigma=i(G W \Gamma-G W)$ have approximately the same magnitude at the HOMO and the LUMO of each molecule. As a function of molecule size, $\Delta \Sigma$ fluctuates around $0.6 \mathrm{eV}$ to $0.7 \mathrm{eV}$ across the family of fullerenes studied, with the exception of $\mathrm{C}_{20}$ where it is around $0.8 \mathrm{eV}$. The "implicit vertex contribution", $i\left(G W-G W_{0}\right)$ is typically five times smaller in magnitude. Self-consistency following the prescription presented here does not modify significantly the strength of vertex contributions. This behavior is similar to the one observed in small oligoacenes (benzene and naphthalene) [15] but it is somewhat different from the scenario observed in silicon nanocrystals. There, the explicit vertex contribution 
TABLE IV: Excitation energy of the first spin-triplet state. Calculated values under the " $\mathrm{GW}_{f^{-}}$ BSE" column were obtained using the $\mathrm{GW}_{f}$ approximation in the electron self-energy. Column

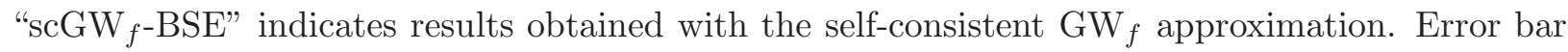
in $\mathrm{QMC}$ values are indicated between parentheses. All energies in $\mathrm{eV}$.

\begin{tabular}{cccccc}
\hline \hline & TDLDA & QMC & GW $_{f}$-BSE & scGW -BSE & Exp. \\
\hline $\mathrm{C}_{20}$ & 0.57 & $0.87(8)$ & -0.12 & 0.62 & \\
$\mathrm{C}_{24}$ & 0.43 & $0.90(14)$ & -0.07 & 0.67 & \\
$\mathrm{C}_{50}$ & 0.20 & $0.61(13)$ & -0.17 & 0.24 & \\
$\mathrm{C}_{60}$ & 1.52 & $2.34(19)$ & 1.14 & 1.65 & $1.58^{a}$ \\
$\mathrm{C}_{70}$ & 1.62 & $2.31(12)$ & 1.01 & 1.45 & $1.55^{b}$ \\
$\mathrm{C}_{80}\left(\mathrm{D}_{5 d}\right)$ & 0.41 & $0.07(10)$ & -0.15 & 0.20 & \\
$\mathrm{C}_{80}\left(\mathrm{I}_{h}\right)$ & 0.11 & $0.26(10)$ & -0.35 & -0.06 & -0.02 \\
\hline average error & 0.01 & 0.76 & -0.49 & 0.09 & \\
root mean square error & 0.05 & 0.76 & 0.49 & \\
\hline \hline
\end{tabular}

${ }^{a}$ Ref. 41. ${ }^{b}$ Ref. 42 .

is no more than around $0.3 \mathrm{eV}$ and cancellation between explicit and implicit contributions is more complete.

Table IV shows the calculated and measured excitation energy of the first spin-triplet electronic states in each fullerene. Experimental data were obtained from phosphorescence decay measurements, and they include a Stokes shift absent in the theoretical calculations. Based on $\triangle$ SCF-DFT calculations, we estimate the Stokes shift to be no more than $0.2 \mathrm{eV}$. Independent evidence for small Stokes shift is found in the photoelectron spectra of $\mathrm{C}_{60}^{-}$ and $\mathrm{C}_{70}^{-}$, which show a high 0-0 line followed by lower vibrational side bands at higher energy [31, 32]. TDLDA gives good excitation energies, with contrasts with the well-known gap underestimation of carbon nanotubes [36] and of periodic systems in general [12]. Selfconsistent GW-BSE is also very accurate, having a discrepancy from experimental data comparable to the Stokes shift. The QMC data is too high by approximately $0.8 \mathrm{eV}$, and the non-self-consistent GW-BSE data is too low by approximately $0.5 \mathrm{eV}$.

The difference in the QMC results must result from a poor cancellation of nodal error between ground and triplet excited states. For small molecules it is possible to improve 
the nodal surface by constructing multideterminant trial wavefunctions or performing additional wavefunction optimization[37], but these techniques are not yet practical for the large fullerenes. Clearly fullerenes represent a good benchmark for methods to improve the nodal surfaces.

The GW-BSE results reflect the discrepancies in the electron affinity: since low-energy optical excitations usually involve the LUMO, excitation energies obtained without selfconsistency are underestimated by approximately $0.6 \mathrm{eV}$ (column "GW $f_{f}$ BSE" in Table V]. We further examine the observed discrepancies by computing higher-energy neutral excitations of $\mathrm{C}_{60}$. Table $\mathrm{V}$ presents excitation energies of the first few spin-singlet states and the projection of each excited state on the subspace of electron transitions from the HOMO to the LUMO. This projection is simply a sum over overlap integrals between the GW-BSE eigenstates $|S\rangle$ and the many-body states obtained by promoting one electron from the HOMO to the LUMO in the ground state $|G\rangle$ :

$$
P_{H-L}=\sum_{j}^{\text {HOMO LUMO }} \sum_{i}\left|\left\langle S\left|a_{i}^{\dagger} a_{j}\right| G\right\rangle\right|^{2}
$$

where $a_{i}^{\dagger}$ and $a_{j}$ are many-body creation and destruction operators respectively. This projection is not readily available in DMC since only the probability density consisting of the product of the QMC ground state and DFT-based trial wavefunction is available.

Identification of the various excitations in $\mathrm{C}_{60}$ is facilitated because the molecule is highly symmetric, leading to high degeneracy and wide energy separation between states. The HOMO and the LUMO of $\mathrm{C}_{60}$ have symmetry representation $\mathrm{h}_{u}$ and $\mathrm{t}_{u}^{1}$ respectively. Electronic transitions between them give rise to four multiplets $\mathrm{T}_{g}^{1}, \mathrm{H}_{g}, \mathrm{~T}_{g}^{2}, \mathrm{G}_{g}$. Table $\mathrm{V}$ shows that the excitation energy of all four multiplets is underestimated within the nonself-consistent $\mathrm{GW}_{f}$ approximation, typically by $0.6 \mathrm{eV}$. This is a direct consequence of the severe overestimation of the electron affinity if self-consistency is not imposed. Some of the higher-energy excitations do not involve the LUMO, such as excitation $\mathrm{T}_{u}^{2}$. For them, the GW-BSE prediction is compatible with experimental data.

Finally, Table IV shows that the most stable fullerenes, namely $\mathrm{C}_{60}\left(\mathrm{I}_{h}\right)$ and $\mathrm{C}_{70}\left(\mathrm{D}_{5 h}\right)$, have the largest triplet excitation energies. Less stable fullerenes have small triplet excitation energy, of the order of $0.4 \mathrm{eV}$, whereas $\mathrm{C}_{80}\left(\mathrm{I}_{h}\right)$ is predicted to have the smallest triplet energy: $0.11 \mathrm{eV}$ (TDLDA). Knowing that triplet excitation energies are related to the energy gap, 
TABLE V: First spin-singlet excitation energies in $\mathrm{C}_{60}$. All energies in $\mathrm{eV}$. We use the same notation of Table IV. Column labeled "H-L" indicates the projection of this excitation onto the HOMO-LUMO subspace (see text). Experimental data from Ref. 43 .

\begin{tabular}{lccccc}
\hline \hline & TDLDA & $\mathrm{GW}_{f}$-BSE & scGW $_{f}$-BSE & $\mathrm{H}-\mathrm{L}$ & Exp. \\
\hline $\mathrm{T}_{g}^{1}$ & 1.69 & 1.47 & 1.88 & $99 \%$ & $1.82-1.97$ \\
$\mathrm{~T}_{g}^{2}$ & 1.71 & 1.53 & 1.92 & $99 \%$ & $1.82-1.97$ \\
$\mathrm{G}_{g}$ & 1.64 & 1.41 & 1.82 & $99 \%$ & $2.00-2.21$ \\
$\mathrm{H}_{g}$ & 1.79 & 1.70 & 2.15 & $94 \%$ & $2.25-2.32$ \\
\hline \hline
\end{tabular}

this trend shows perfectly correlation between stability and energy gap: stable fullerenes have wide gap between HOMO and LUMO, unstable fullerenes have narrow gap between HOMO and LUMO.

\section{CONCLUSION}

We have calculated and analyzed the low energy excitations of fullerenes $\mathrm{C}_{20}$ to $\mathrm{C}_{80}$ using three theoretical methods: density functional theory, many-body GW-BSE and diffusion Quantum Monte Carlo. Overall these methods give an accurate description of the optical properties. Ionization potentials are approximately constant and well predicted by all methods. The more stable fullerenes $\left(\mathrm{C}_{60}\right.$ and $\left.\mathrm{C}_{70}\right)$ are found to have highest excitation energy. Stability correlates well with energy gap. We find a weak dependence of electron affinity with molecule size, with important implications for doping and functionalization of fullerenes. We find that two ingredients are essential to bring GW-BSE predictions to agree with experimental data: vertex corrections, which are responsible for an almost rigid shift of electronic orbitals with respect to the vacuum level, and self-consistency, which widens the energy gap between occupied orbitals and unoccupied orbitals. QMC calculations of the lowest energy triplet excitation using a single determinant of Kohn-Sham orbitals give results higher than experiment by approximately $0.8 \mathrm{eV}$, indicating that the DFT-derived trial wave-functions presumably give nodal errors of that magnitude. Therefore while QMC calculation of even larger systems are possible as the systems size increases so does the nodal error. Methods to improve the nodal structure are required to achieve a total energy 
resolution of $0.1 \mathrm{eV}$. We believe the above observations will guide method selection for excitations in nanostructures. Clearly, the robust design of optical nanoarchitectures will require further developments in many-body theories such as GW-BSE and QMC. We also encourage more precise experimental determination of electronic excitations in well-characterized nanostructures to provide a more severe test to theory.

Research performed at the Materials Science and Technology Division, sponsored by the Division of Materials Sciences Engineering BES, U.S. DOE, and at the Center for Nanophase Materials Sciences, sponsored by the Division of Scientific User Facilities, U.S. Department of Energy under contract with UT-Battelle, LLC. Work at the Lawrence Livermore National Laboratory was performed under the auspices of the U.S. Department of Energy under Contract DE-AC52-07NA27344. Computational support was provided by the Texas Advanced Computing Center (TACC) and the National Energy Research Scientific Computing Center (NERSC) and Lawrence Livermore National Laboratory.

[1] M. S. Dresselhaus, G. Dresselhaus, and P. C. Eklund, Science of fullerenes and carbon nanotubes: their propertiions (Academic Press, San Diego, 1996).

[2] See EPAPS Document No. ${ }^{* * * * * *}$ for atomic coordinates of the molecules analyzed in this work. This document can be reached through a direct link in the online article's HTML reference section or via the EPAPS homepage (http://www.aip.org/pubservs/epaps.html).

[3] E. Osawa, Perspectives of Fullerene Nanotechnology (Springer Verlag, Berlin, 2002).

[4] J. G. Xue, S. Uchida, B. P. Rand, and S. R. Forrest, Appl. Phys. Lett. 84, 3013 (2004).

[5] F. Yang, M. Shtein, and S. R. Forrest, J. Appl. Phys. 98, 014906 (2005).

[6] R. Pfeiffer, M. Holzweber, H. Peterlik, H. Kuzmany, Z. Liu, K. Suenaga, and H. Kataura, Nano Lett. 7, 2428 (2007).

[7] E. Hernandez, V. Meunier, B. W. Smith, R. Rurali, H. Terrones, M. B. Nardelli, M. Terrones, D. E. Luzzi, and J. C. Charlier, Nano Lett. 3, 1037 (2003).

[8] C. Rutherglen and P. Burke, Nano Lett. 7, 3296 (2007).

[9] J. B. Neaton, M. S. Hybertsen, and S. G. Louie, Phys. Rev. Lett. 97, 216405 (2006).

[10] V. Barone, J. E. Peralta, M. Wert, H. J., and G. Scuseria, Nano Lett. 5, 1621 (2005).

[11] C. Spataru, S. Ismail-Beigi, B. L.X., and L. SG, Phys. Rev. Lett. 92, 077402 (2004). 
[12] G. Onida, L. Reining, and A. Rubio, Rev. Mod. Phys. 74, 601 (2002).

[13] W. Aulbur, L. Jönsson, and J. Wilkins, Solid State Physics (Academic Press, New York, 2000), vol. 54, p. 1.

[14] W. M. C. Foulkes, L. Mitas, R. J. Needs, and G. Rajagopal, Rev. Mod. Phys. 73, 33 (2001).

[15] M. L. Tiago and J. R. Chelikowsky, Phys. Rev. B 73, 205334 (2006).

[16] A. J. Williamson, J. C. Grossman, R. Q. Hood, A. Puzder, and G. Galli, Phys. Rev. Lett. 89, $196803(2002)$.

[17] M. Rohlfing and S. G. Louie, Phys. Rev. B 62, 4944 (2000).

[18] J. A. Zimmerman, J. R. Eyler, S. B. H. Bach, and S. W. McElvany, J. Chem. Phys. 94, 3556 (1991).

[19] J. P. Perdew, K. Burke, and M. Ernzerhof, Phys. Rev. Lett. 77, 3865 (1996).

[20] R. W. Martin, Electronic structure : basic theory and practical methods (Cambridge University Press, Cambridge, UK, 2004).

[21] M. L. D. Puerto, M. L. Tiago, and J. R. Chelikowsky, Phys. Rev. Lett. 97, 096401 (2006).

[22] R. Needs, M. Towler, N. Drummond, and P. L. Ríos, CASINO version 2.1 User Manual (University of Cambridge, Cambridge, 2007).

[23] M. Casula, Phys. Rev. B 74, 161102 (2006).

[24] W. M. C. Foulkes, R. Q. Hood, and R. J. Needs, Phys. Rev. B 60, 4558 (1999).

[25] N. D. Drummond, A. J. Williamson, R. J. Needs, and G. Galli, Phys. Rev. Lett. 95, 096801 (2005).

[26] F. Furche and R. Ahlrichs, J. Chem. Phys. 114, 10362 (2001).

[27] R. Bauernschmitt, R. Ahlrichs, F. H. Henrich, and M. M. Kappes, J. Am. Chem. Soc. 120, 5052 (1998).

[28] L. E. Brus, J. Chem. Phys. 79, 5566 (1983).

[29] G. Seifert, K. Vietze, and R. Schmidt, J. Phys. B-Atomic Molec. Optical Phys. 29, 5183 (1996).

[30] D. L. Lichtenberger, K. W. Nebesny, C. D. Ray, D. R. Huffman, and L. D. Lamb, Chem. Phys. Lett. 176, 203 (1991).

[31] X. B. Wang, C. F. Ding, and L. S. Wang, J. Chem. Phys. 110, 8217 (1999).

[32] X. B. Wang, H. K. Woo, X. Huang, M. M. Kappes, and L. S. Wang, Phys. Rev. Lett. 96, 143002 (2006). 
[33] A. J. Morris, M. Stankovski, K. T. Delaney, P. Rinke, P. García-González, and R. W. Godby, Phys. Rev. B 76, 155106 (2007).

[34] T. Kotani and M. van Schilfgaarde, Phys. Rev. B 76, 165106 (2007).

[35] S. Ismail-Beigi and S. G. Louie, Phys. Rev. Lett. 90, 076401 (2003).

[36] S. M. Bachilo, M. S. Strano, C. Kittrell, R. H. Hauge, R. E. Smalley, and R. B. Weisman, Science 298, 2361 (2002).

[37] C. J. Umrigar, J. Toulouse, C. Filippi, S. Sorella, and R. G. Hennig, Phys. Rev. Let. 99, $179902(2007)$.

[38] D. L. Lichtenberger, M. E. Rempe, and S. B. Gogosha, Chem. Phys. Lett. 198, 454 (1992).

[39] H. Prinzbach, F. Wahl, A. Weller, P. Landenberger, J. Wörth, L. T. Scott, M. Gelmont, D. Olevano, F. Sommer, and B. von Isendorff, Chemistry-A European Journal 12, 6268 (2006).

[40] S. H. Yang, J. Conceicao, O. Cheshnovsky, and R. E. Smalley, Chem. Phys. Lett. 139, 233 (1987).

[41] M. G. Giuffreda, F. Negri, and G. Orlandi, J. Phys. Chem. A 105, 9123 (2001).

[42] J. B. M. Warntjes, I. Holleman, G. Meijer, and E. J. J. Groenen, Chem. Phys. Lett. 261, 495 (1996).

[43] S. Leach, M. Vervloet, A. Despres, E. Breheret, J. P. Hare, T. J. Dennis, H. W. Kroto, R. Taylor, and D. R. M. Walton, Chem. Phys. 160, 451 (1992).

[44] QMC statistical errors are $0.1 \mathrm{eV}$ to $0.2 \mathrm{eV}$. All GW results have estimated systematic convergence errors of less than $0.2 \mathrm{eV}$. 

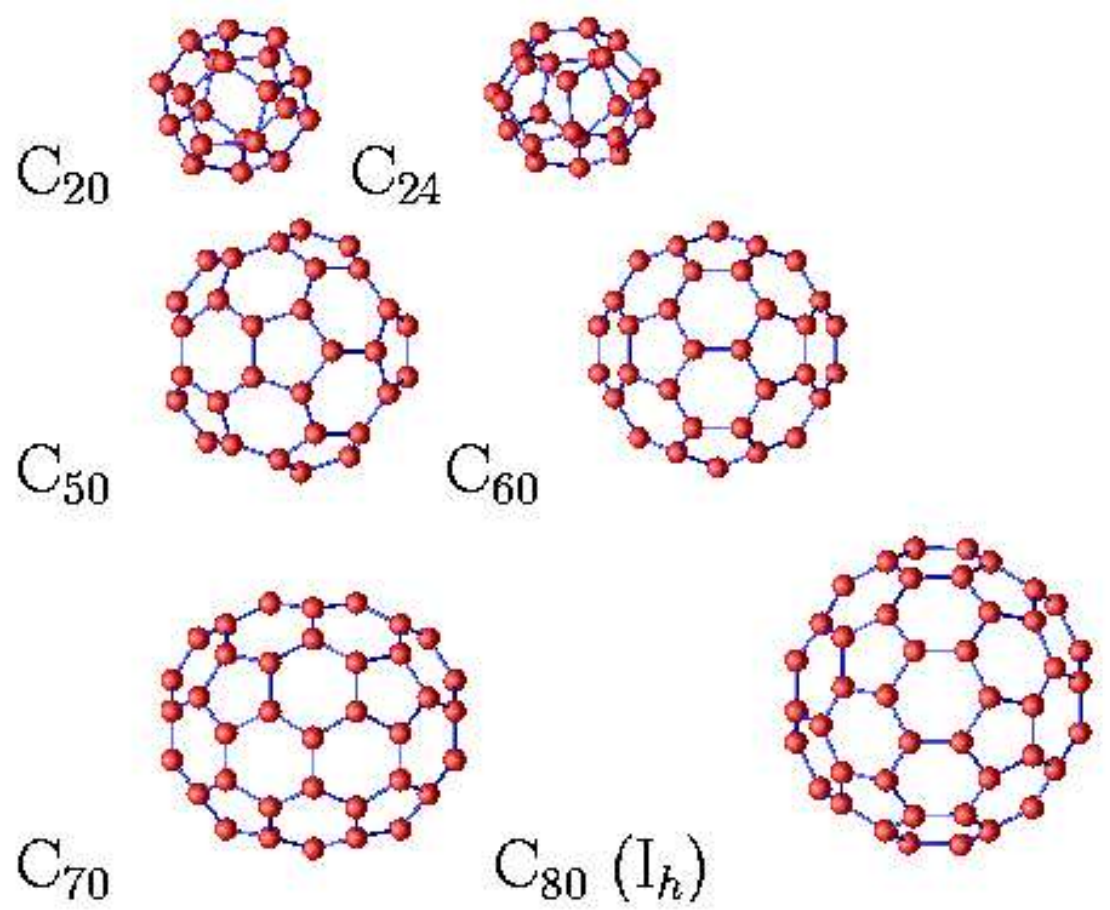

$\mathrm{C}_{80}\left(\mathrm{D}_{5 d}\right)$

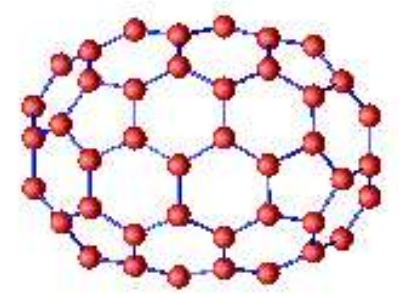

FIG. 1: (Color online) Structures of the fullerenes with corresponding point group representations in parentheses: $\mathrm{C}_{20}\left(\mathrm{I}_{h}\right), \mathrm{C}_{24}\left(\mathrm{D}_{6 d}\right), \mathrm{C}_{50}\left(\mathrm{D}_{5 h}\right), \mathrm{C}_{60}\left(\mathrm{I}_{h}\right), \mathrm{C}_{70}\left(\mathrm{D}_{5 h}\right), \mathrm{C}_{80}\left(\mathrm{I}_{h}\right), \mathrm{C}_{80}\left(\mathrm{D}_{5 d}\right)$. 